\title{
Choosing sets: preface to the special issue on set optimization and applications
}

\author{
Andreas H Hamel ${ }^{1}$. Andreas Löhne² \\ Published online: 1 January 2020 \\ ๑) Springer-Verlag GmbH Germany, part of Springer Nature 2020
}

In his 1979 Econometrica paper Kreps (1979), David M. Kreps discussed the following example.

Suppose you want to go to a restaurant for dinner, and all what counts for you is the quality of the meals. Suppose further that there are two restaurants in town, and by looking at their menus A and B on your smartphone you realize that for each meal on A there is one on B which you like better (or at least as much as the one on A). In which restaurant would you reserve a dinner table?

Formally, the relation between the two menus can be written as

$$
\forall a \in A, \exists b \in B: b \succeq a
$$

where $\succeq$ indicates your preference for (single) meals which is assumed to be a reflexive and transitive relation on the set of meals on any restaurant menu. In Kreps (1979), this preference relation was also assumed to be total.

This dinner decision process has a two stage character. In the first stage one chooses a menu (i.e., a restaurant), and in the second stage, only later, a dish from the chosen menu. While the latter should clearly be a maximal element from that menu with respect to the preference $\succeq$, it could be argued that the choice of the restaurant should be made according to the relation defined by (1). Such a relation is nowadays called a set relation, and it is one of two possiblities to extend a reflexive and transitive relation $\succeq$ on a set to a reflexive and transitive relation on its power set. The other one is

$$
\forall b \in B, \exists a \in A: b \succeq a
$$

$凶$ Andreas H Hamel

andreas.hamel@unibz.it

Andreas Löhne

andreas.loehne@uni-jena.de

1 Faculty of Economics and Management, Free University of Bozen-Bolzano, Bolzano, Italy

2 Faculty of Mathematics and Computer Science, Friedrich Schiller University Jena, Jena, Germany 
with the following interpretation: for each meal on the menu B there is one on A which you like less or at most as much as the one on $\mathrm{B}$. The reader may check that the two relations defined by (1) and (2) do not coincide in general, even if $\succeq$ is total (which is not assumed here).

It is remarkable (and not so well-known) that such set relations have been introduced "intuitively" in economics already some 20 years before they started to gain popularity in the (mathematical) vector optimization community through the publications of D. Kuroiwa and coauthors, e.g., Kuroiwa et al. (1997). For more references, compare Hamel et al. (2015).

Several questions arise. First, which of the two set relations should be used for a particular decision? Recently, several more set relations have been introduced, see, e.g., Jahn and Truong (2011), Chen et al. (2017) which makes the question even more pressing. However, it seems obvious that relation (1) is much more appropriate for the menu decision than relation (2). Secondly, what should be understood by a solution of an optimization problem with respect to the order (1) or (2)? And then, how can a corresponding set optimization theory be developed? An early attempt to deal with these questions is Alonso and Rodríguez-Marín (2005), one of the first versions of Ekeland's principle with set relations can be found in Truong (2005).

In 1998, MMOR published a special issue on set-valued optimization Chen and Jahn (1998). Its contributions were not driven by particular applications (in economics, for example), and they did not discuss or apply set relations. However, only a few years later (Jahn 2004, p. 378), one of the editors of this SI wrote that the set relation approach 'opens a new and wide field of research' and set relations 'turn out to be very promising in set optimization.' In our opinion, this was an excellent prophecy, and the current special issue shows how topics and approaches have changed over the last 20 years.

The set relation approach was extended to the "complete lattice approach" which resolved the issue of the missing infimum/supremum in vector optimization, see, e.g., Löhne (2011). The basic observation is that the supremum with respect to the relation (1) as well as the infimum with respect to the one in (2) are meaningful concepts-in contrast to infima and suprema with respect to vector orders. A complete duality theory for set- (and also vector-valued) functions could be established including satisfying duality results for multicriteria linear optimization Heyde et al. (2009). New applications came within reach (see Hamel et al. (2015) for an overview): for example, the complete lattice-valued duality approach produced consistent pricing processes as dual variables for financial market models with frictions Hamel et al. (2011) — exactly the "right" dual variables which were used in finance before Jouini et al. (2004), Kabanov (1999); new solution concepts for games with multi-dimensional payoffs Hamel and Löhne (2018) as well as quantiles for multivariate random variables Hamel and Kostner (2018) could be defined in ways which parallel the one-dimensional case much more than all previous approaches.

The infima and suprema with respect to set relations essentially coincide with unions and intersections. One may ask how these concepts are interpreted in applications. This question points toward best and worst case analysis. The supremum with respect to the relation (1) in the restaurant example above corresponds to the set of all possible dishes. If the "base relation" $\succeq$ is total, then it is sufficient to know the best dish of the 
union of all menus (if $\succeq$ is not antisymmetric there might even be more than one). If $\succeq$ is not total, then it is not so clear what the best case is: there might be a variety of dishes in different restaurants which are not dominated by any other dish.

In this situation, the first stage decision is about finding menus which are not dominated by others with respect to the relation (1): restaurants with dominated menus will not be taken into consideration for a dinner reservation. On the other hand, it would be good to know all the options, i.e., the union of all menus where the dominated menus could be left out - this union corresponds to the supremum with respect to the relation (1).

Therefore, the solution concept for set optimization problems proposed in Heyde and Löhne (2011) includes the infimum (or supremum) as well as non-dominated sets. On the other hand, one can find examples where the supremum can be generated by dominated sets which shows that minimality/maximality (= not dominated with respect to minimization and maximization, respectively) on the one hand and attainment of the infimum/supremum on the other hand become different concepts in complete lattice-valued optimization. For games with multi-dimensional payoffs, these concepts correspond to worst-case (=maximin or minimax) approaches and are different from more traditional Nash equilibrium type concepts Hamel and Löhne (2018), Maeda (2015). It turned out that this approach is especially useful if the underlying "base" preference/order relation is not total-in contrast to Kreps' assumption.

Surprisingly, for a long time mathematics has contributed very little to the theory of set relations and its applications. We think that the development only just started, and many interesting new questions as well as applications are already under discussion or will appear in the near future.

In this Special Issue, one can find a variety of topics and results related to set optimization and its applications.

Baes and Munari discuss features of risk minimizing mappings which appear within the context of set-valued risk measures in finance and Crespi and Mastrogiacomo investigate the asymptotic behaviour of estimators of such set-valued risk measures.

The paper by Weißing addresses the problem to compute the projection of a polyhedral convex set. This polyhedral projection problem provides a fundamental computational tool for a calculus of sets and for set optimization.

In Kostner's paper, a set optimization approach to statistics is turned into a tool for multi-criteria decision making. The special feature is the inclusion of several decision makers who may have different opinions.

The contribution of Jiménez et al. investigates two set scalarization functions, which are extensions of the Hiriart-Urruty oriented distance function. The functions are used to characterize minimality with respect to set relations.

The article by Ansari et al. deals with a set-valued Ekeland's variational principle and equivalent variants. The results are based on weighted set relations, which are mixtures of the upper and lower set relation of the type (1), (2) above.

The paper by Truong introduces a concept of slopes for set-valued maps based on scalarization. Applications to set optimization are error bounds for lower level sets and the existence of weak minimizers under a Palais-Smale type condition. 
The concepts in the paper by Lepinette could be seen as an alternative to set optimization when it comes to supremum/infimum definitions for set-valued stochastic optimization within the framework of random sets.

In Han et al., the Painlevé-Kuratowski convergence of sets of $\varepsilon$-minimizers to minimizers is studied as well as extended well-posedness of set optimization problems.

Last, but by no means least, we would like to express our gratitude to the MMOR team as well as to the authors for making this SI possible. A special "Thank You!" goes to the many reviewers who often contributed to one or even more rounds of revisions with many suggestions and detailed remarks_-we very much appreciate this effort!

\section{References}

Alonso M, Rodríguez-Marín L (2005) Set-relations and optimality conditions in set-valued maps. Nonlinear Anal 63(8):1167-1179

Chen GY, Jahn J (1998) Special issue on set-valued optimization. Math Methods Oper Res 48(2):151-152

Chen JW, Köbis E, Köbis M, Yao J-C (2017) A new set order relation in set optimization. J Nonlinear Convex Anal 18(4):637-649

Hamel AH, Heyde F, Löhne A, Rudloff B, Schrage C (2015) Set optimization-a rather short introduction. In: Hamel AH, Heyde F, Löhne A, Rudloff B, Schrage C (eds) Set optimization and applications-the state of the art. From set relations to set-valued risk measures. Springer, Berlin, pp 65-141

Hamel AH, Heyde F, Rudloff B (2011) Set-valued risk measures for conical market models. Math Financ Econ 5:1-28

Hamel AH, Löhne A (2018) A set optimization approach to zero-sum matrix games with multi-dimensional payoffs. Math Methods Oper Res 88(3):369-397

Hamel AH, Kostner D (2018) Cone distribution functions and quantiles for multivariate random variables. J Multivar Anal 167:97-113

Heyde F, Löhne A (2011) Solution concepts for vector optimization problems: a fresh look at an old story. Optimization 60(12):1421-1440

Heyde F, Löhne A, Tammer C (2009) Set-valued duality theory for multiple objective linear programs and application to mathematical finance. Math Methods Oper Res 69(1):159-179

Jahn J (2004) Vector optimization. Springer, Berlin

Jahn J, Truong XDH (2011) New order relations in set optimization. J Optim Theory Appl 148(2):209-236

Jouini E, Meddeb M, Touzi N (2004) Vector-valued coherent risk measures. Finance Stoch 8(4):531-552

Kabanov YM (1999) Hedging and liquidation under transaction costs in currency markets. Finance Stoch 3(2):237-248

Kuroiwa D, Tanaka T, Truong XDH (1997) On cone convexity of set-valued maps. Nonlinear Anal 30(3):1487-1496

Kreps DM (1979) A representation theorem for "preference for flexibility". Econometrica 47(3):565-577

Löhne A (2011) Vector optimization with infimum and supremum. Springer, Berlin

Maeda T (2015) On characterization of Nash equilibrium strategy in bi-matrix games with set payoffs. In: Hamel AH, Heyde F, Löhne A, Rudloff B, Schrage C (eds) Set optimization and applications-the state of the art. From set relations to set-valued risk measures. Springer, Berlin, pp 313-331

Truong XDH (2005) Some variants of the Ekeland variational principle for a set-valued map. J Optim Theory Appl 124(1):187-206

Publisher's Note Springer Nature remains neutral with regard to jurisdictional claims in published maps and institutional affiliations. 